\title{
CALCULATION OF HEAT TRANSFER COEFFICIENTS FOR FOODS
}

\author{
by \\ Brian A. Fricke, Ph.D. \\ Visiting Assistant Professor \\ and \\ Bryan R. Becker, Ph.D., P.E. \\ Professor \\ Mechanical Engineering \\ University of Missouri-Kansas City \\ 5100 Rockhill Road \\ Kansas City, MO 64110-2499
}

25 May 2002 


\title{
CALCULATION OF HEAT TRANSFER COEFFICIENTS FOR FOODS
}

\author{
Brian A. Fricke, Ph.D. \\ Mechanical Engineering \\ University of Missouri-Kansas City \\ 5100 Rockhill Road, Kansas City, MO 64110-2499 \\ Bryan R. Becker, Ph.D., P.E. \\ Mechanical Engineering \\ University of Missouri-Kansas City \\ 5100 Rockhill Road, Kansas City, MO 64110-2499
}

\begin{abstract}
The design of food refrigeration equipment requires estimation of the cooling and freezing times of foods and beverages, as well as the corresponding refrigeration loads. The accuracy of these estimates, in turn, depends upon accurate estimates of the surface heat transfer coefficient for the cooling or freezing operation. A unique iterative algorithm, utilizing the concept of "equivalent heat transfer dimensionality," was developed to obtain heat transfer coefficients from cooling curves. Cooling curves were obtained from an industrial survey, and, utilizing the iterative algorithm, heat transfer coefficients were determined for various food items. A small portion of that data presented in this paper. This information will make possible a more accurate determination of cooling and freezing times and corresponding refrigeration loads.
\end{abstract}

\section{$\underline{\text { Introduction }}$}

In many food processing applications, including cooling and freezing, transient convective heat transfer occurs between a fluid medium and the solid food item [1]. Knowledge of the surface heat transfer coefficient is required to design the equipment wherein convection heat transfer is used to process foods and beverages. Newton's law of cooling defines the surface heat transfer coefficient, $h$, as follows:

$$
q=h A\left(t_{s}-t_{m}\right)
$$

A small number of studies have been performed to measure or estimate the surface heat transfer coefficient during cooling, freezing or heating of food items [1-34]. In addition, a detailed literature survey 
has been compiled by Arce and Sweat [35]. These studies present surface heat transfer coefficient data and correlations for only a very limited number of food items and process conditions.

Thus, there was clearly a need to expand upon the previous work by developing a comprehensive database of heat transfer coefficients for a wide range of food items and process conditions. Hence, the objective of this study was to determine the surface heat transfer coefficients for a wide variety of foods during cooling and freezing processes.

\section{Existing Techniques to Determine the Surface Heat Transfer Coefficients of Foods}

One method for obtaining the surface heat transfer coefficient of a food product with an internal temperature gradient involves the use of cooling curves. For simple, one-dimensional food geometries such as infinite slabs, infinite circular cylinders or spheres, there exists empirical and analytical solutions to the one-dimensional transient heat equation. The slope of the cooling curve may be used in conjunction with these solutions to obtain the Biot number for the cooling process. The heat transfer coefficient may then be determined from the Biot number.

All cooling processes exhibit similar behavior. After an initial "lag", the temperature at the thermal center of the food item decreases exponentially [36]. A cooling curve depicting this behavior can be obtained by plotting, on semi-logarithmic axes, the fractional unaccomplished temperature difference versus time. The fractional unaccomplished temperature difference, $Y$, is defined as follows:

$$
Y=\frac{t_{m}-t}{t_{m}-t_{i}}=\frac{t-t_{m}}{t_{i}-t_{m}}
$$

This semi-logarithmic temperature history curve consists of one initial curvilinear portion, followed by one or more linear portions. Simple empirical formulae, which model this cooling behavior, have been proposed for estimating the cooling time of foods and beverages. These models incorporate two factors, $f$ and $j$, which represent the slope and intercept, respectively, of the semi-logarithmic temperature history curve.

The $j$ factor is a measure of the "lag" between the onset of cooling and the exponential decrease in the temperature of the food. The $f$ factor represents the time required to obtain a $90 \%$ reduction in the nondimensional temperature difference. The $f$ factor is a function of the Biot number while the $j$ factor is a function of the Biot number and the location within the food item.

The general form of the cooling time model is:

$$
Y=j e^{-2.303 \theta / f}
$$


For simple geometrical shapes, such as infinite slabs, infinite circular cylinders and spheres, the simple empirical cooling time model given by Equation (3) can be derived analytically, and thus, analytical expressions can be derived for the $f$ and $j$ factors. The one-dimensional transient heat equation may be written as follows for infinite slabs, infinite circular cylinders and spheres:

$$
\left(\frac{1}{z^{a}}\right)\left(\frac{\partial}{\partial z}\right)\left[z^{a}\left(\frac{\partial \phi}{\partial z}\right)\right]=\left(\frac{1}{\alpha}\right)\left(\frac{\partial \phi}{\partial \theta}\right)
$$

where $a$ is the value 0,1 , or 2 , for slabs, cylinders or spheres, respectively.

The initial and boundary conditions are as follows:

$$
\begin{gathered}
\phi(z, 0)=\left(t_{i}-t_{m}\right) \\
\frac{\partial}{\partial z} \phi(0, \theta)=0 \\
-k\left(\frac{\partial}{\partial z} \phi(Z, \theta)\right)+h \phi(Z, \theta)=0
\end{gathered}
$$

For infinite slabs, $Z$ is the half thickness of the slab. For infinite cylinders and spheres, $Z$ is the radius of the cylinder or sphere.

In order to non-dimensionalize the solutions of Equation (4), two dimensionless parameters are introduced, namely, the Biot number and the Fourier number. The Biot number is defined as follows:

$$
B i=\frac{h Z}{k}
$$

and the Fourier number is defined as follows:

$$
F_{O}=\frac{\alpha \theta}{Z^{2}}
$$

The infinite series solution for the dimensionless center temperature distribution for Equation (4) is given by Carslaw and Jaeger [37] as follows:

$$
Y=\sum_{n=1}^{\infty} A_{n} B_{n}
$$

Here, $A_{n}$ is given in Table 1. 


\section{TABLE 1}

Equations for $A_{n}$

\begin{tabular}{ccc}
\hline Infinite Slab & Infinite Cylinder & Sphere \\
\hline$A_{n}=\frac{2 \sin \mu_{n}}{\mu_{n}+\sin \mu_{n} \cos \mu_{n}}$ & $A_{n}=\frac{2 B i}{\mu_{n}^{2}+B i^{2}+\mathrm{J}_{o}\left(\mu_{n}\right)}$ & $A_{n}=\frac{2 B i \sin \mu_{n}}{\mu_{n}-\sin \mu_{n} \cos \mu_{n}}$
\end{tabular}

For all three shapes, $B_{n}$ is given as:

$$
B_{n}=\exp \left(-\mu_{n}^{2} F_{O}\right)
$$

Here, $\mu_{n}$ is a parameter specified by a characteristic equation which is geometry dependent.

After the initial "lag" period has passed, in which case $F_{O} \geq 0.2$, the second and higher terms of Equation (10) are assumed to be negligible [21]. Thus, Equation (10) can be simplified as follows:

$$
Y=A_{1} B_{1}
$$

Here, $A_{1}$ is given in Table 2.

TABLE 2

Equations for $A_{1}$

\begin{tabular}{ccc}
\hline Infinite Slab & Infinite Cylinder & Sphere \\
\hline$A_{1}=\frac{2 \sin \mu_{1}}{\mu_{1}+\sin \mu_{1} \cos \mu_{1}}$ & $A_{1}=\frac{2 B i}{\mu_{1}^{2}+B i^{2}+\mathrm{J}_{\mathrm{o}}\left(\mu_{1}\right)}$ & $A_{1}=\frac{2 B i \sin \mu_{1}}{\mu_{1}-\sin \mu_{1} \cos \mu_{1}}$ \\
\hline
\end{tabular}

For all three shapes, $B_{1}$ is given as:

$$
B_{1}=\exp \left(-\mu_{1}^{2} F o\right)
$$

The characteristic equations for $\mu_{1}$ are given in Table 3 .

Thus, by comparing Equations (3) and (12), Dincer [21] showed that:

$$
\begin{gathered}
j=A_{1} \\
\frac{2.303 \theta}{f}=\mu_{1}^{2} F_{O}
\end{gathered}
$$


TABLE 3

Characteristic Equations for $\mu_{1}$

\begin{tabular}{ccc}
\hline Infinite Slab & Infinite Cylinder & Sphere \\
\hline $\cot \mu_{1}=\frac{\mu_{1}}{B i}$ & $\frac{\mathrm{J}_{\mathrm{o}}\left(\mu_{1}\right)}{\mathrm{J}_{1}\left(\mu_{1}\right)}=\frac{\mu_{1}}{B i}$ & $\cot \mu_{1}=\frac{1-B i}{\mu_{1}}$ \\
\hline
\end{tabular}

Since the Fourier number, $F o$, of a cooling process can be readily determined, and, provided that the value of $f$ can be determined from a cooling curve, the value of $\mu_{1}$ can be obtained by rearranging Equation (15):

$$
\mu_{1}=\sqrt{\frac{2.303 \theta}{f F o}}
$$

Then, the Biot number, $B i$, can be obtained from the appropriate equation given in Table 3, depending upon the food geometry. Finally, the surface heat transfer coefficient, $h$, may be obtained through algebraic manipulation of the definition of the Biot number, Equation (8).

\section{Iterative Technique for Determine Heat Transfer Coefficients of Irregularly Shaped Food Items}

The techniques of Dincer, discussed above, are strictly applicable to only regularly shaped food items. Therefore, a unique iterative method was developed to handle irregularly shaped food items. This method utilizes a shape factor called the "equivalent heat transfer dimensionality," developed by Cleland and Earle [38], to extend the applicability of Dincer's techniques to irregularly shaped food items.

The "equivalent heat transfer dimensionality" compares the total heat transfer to the heat transfer through the shortest dimension. Cleland and Earle [38] developed an expression for estimating the equivalent heat transfer dimensionality of irregularly shaped food items as a function of Biot number. However, the cooling time estimation method developed by Cleland and Earle, which incorporates the equivalent heat transfer dimensionality, requires the use of a nomograph. Therefore, Lin et al. [39, 40, 41] expanded upon the work of Cleland and Earle [38] to eliminate the need for a nomograph.

The cooling time of a food is estimated by a first term approximation to the analytical solution for convective cooling of a sphere $[39,40,41]$ :

$$
Y=j \exp \left[\frac{-\omega^{2} k E \theta}{3 \rho c Z^{2}}\right]
$$

where $\omega$ is the first root of the following transcendental function:

$$
\omega \cot \omega+B i-1=0
$$


In Equation (17), the equivalent heat transfer dimensionality, $E$, is given as a function of Biot number, $B i$. Lin et al. [39, 40, 41] present equations for determining the equivalent heat transfer dimensionality for regularly and irregularly shaped food items.

The current iterative method utilizes the cooling coefficient, $C$, which represents minus the slope of the cooling curve:

$$
C=\frac{\omega^{2} k E}{3 \rho c Z^{2}}=\frac{2.303}{f}
$$

Solving Equation (19) for $\omega$ yields the following:

$$
\omega=\sqrt{\frac{3 C Z^{2}}{\alpha E}}
$$

After determining the cooling coefficient, $C$, from the slope of the cooling curve, the value of $\omega$ is obtained from Equation (20) and then substituted into Equation (18) to obtain the Biot number. Note that the equivalent heat transfer dimensionality, $E$, which is a function of Biot number, appears in Equation (20), and thus, iteration of Equations (18) and (20) is required to obtain the Biot number. Once the Biot number has been determined, the heat transfer coefficient can be calculated from the definition of the Biot number, Equation (8).

\section{Cooling and Freezing Curves}

Members of the food refrigeration industry were contacted to collect cooling and freezing curves and surface heat transfer data for various food items. These collected cooling and freezing curves were digitized and a database was developed which contains the digitized temperature versus time data obtained from these curves. The temperatures were non-dimensionalized according to Equation (2) and the natural logarithm of these non-dimensional temperatures were taken. The slopes of the linear portion(s) of the logarithmic temperature versus time data were determined using the linear least-squares-fit technique. These slopes were then used in conjunction with the techniques described in the previous section to determine the heat transfer coefficients for the food items.

\section{$\underline{\text { Calculated Heat Transfer Coefficients }}$}

To illustrate the data which was obtained from the iterative algorithm, a sample of calculated heat transfer coefficients, for cheese, is given in Table 4. This table lists the heat transfer coefficients for the cheese with a description of the packaging, dimensions, and weight, as well as the air temperature and air velocity used to cool or freeze the food items. 
TABLE 4

Calculated Heat Transfer Coefficients for Cheese

\begin{tabular}{|c|c|c|c|c|c|c|c|c|}
\hline 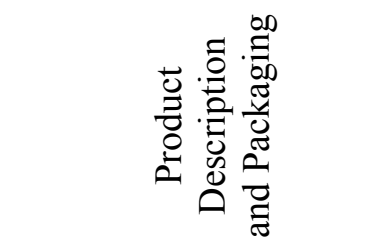 & 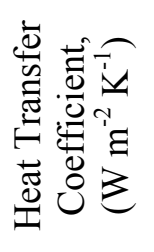 & 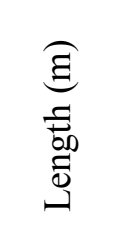 & 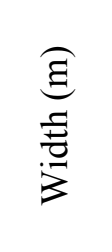 & $\begin{array}{l}\overparen{\Xi} \\
\stackrel{\Xi}{E} \\
\frac{.00}{0} \\
\frac{0}{1}\end{array}$ & 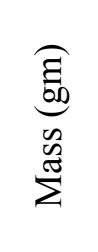 & 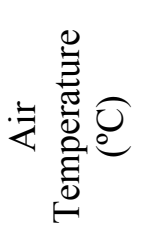 & 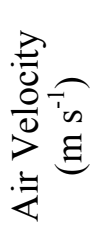 & 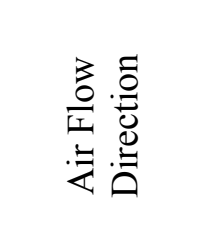 \\
\hline \multirow{3}{*}{ Mozzarella Cheese } & 7.59 & 0.267 & 0.105 & & 2268 & 0.6 & 3. & \multirow{3}{*}{$\begin{array}{l}\text { along length } \\
\text { along length }\end{array}$} \\
\hline & 6.48 & 0.552 & 0.191 & 0.095 & 9072 & -23.3 & 3. & \\
\hline & 7.04 & 0.552 & 0.191 & 0.095 & 9072 & -34.4 & 3. & \\
\hline Cheese Sauce in Pouch & 3.15 & 0.18 & 0.1 & 0.06 & 260 & 2. & 3. & along height \\
\hline $\begin{array}{l}\text { Bacon Cheese in Paper } \\
\text { Tray with Plastic Lid }\end{array}$ & 5.1 & 0.13 & 0.09 & 0.04 & 110 & -5 & 3. & along height \\
\hline \multirow{3}{*}{$\begin{array}{l}\text { Soft Cheese in Hard } \\
\text { Plastic Box with Lid }\end{array}$} & 16.8 & 0.124 & 0.088 & 0.034 & 200 & -10. & 3. & \multirow{3}{*}{$\begin{array}{l}\text { along height } \\
\text { along height } \\
\text { along height }\end{array}$} \\
\hline & 26.5 & 0.124 & 0.088 & 0.051 & 400 & -10 & 3. & \\
\hline & 14.1 & 0.125 & 0.062 & 0.027 & 110 & -5 & 3. & \\
\hline
\end{tabular}

\section{$\underline{\text { Nusselt-Reynolds Correlations }}$}

Non-dimensional analyses were performed to obtain simple heat transfer coefficient correlations which can be used to predict the heat transfer coefficients of food items. A linear least-squares-fit was performed on Nusselt Number versus Reynolds Number data to obtain linear a Nusselt-Reynolds correlation. For this correlation, the Nusselt number, $\mathrm{Nu}$, is defined as:

$$
N u=\frac{h d}{k_{m}}
$$

The Reynolds number, $R e$, is defined as:

$$
\operatorname{Re}=\frac{\rho_{m} U d}{\mu_{m}}
$$

The resulting Nusselt-Reynolds correlation for cheese is as follows:

$$
N u=17.01+0.000575 R e \text { for } 5000<R e<30000
$$

Figure 1 shows the heat transfer coefficient data and the resulting Nusselt-Reynolds correlation for cheese.

\section{$\underline{\text { Conclusions }}$}

An iterative algorithm was developed to estimate the surface heat transfer coefficients of irregularly shaped food items based upon their cooling and freezing curves. This algorithm utilizes the concept of "equivalent heat transfer dimensionality" to extend to irregularly shaped food items existing techniques for 




FIG. 1

Nusselt-Reynolds correlation for cheese.

the calculation of the surface heat transfer coefficient, previously applicable to only regularly shaped food items.

Making use of this algorithm, heat transfer coefficients for various food items were calculated from cooling and freezing curves collected from members of the food refrigeration industry. A sample of the calculated heat transfer coefficient data was presented in this paper for cheese. In addition, a NusseltReynolds correlation was developed to summarize the heat transfer coefficient data for cheese.

\section{Nomenclature}

\begin{tabular}{|c|c|c|c|}
\hline$a$ & geometry factor & $h$ & heat transfer coefficient $\left(\mathrm{W} \mathrm{m}^{-2} \mathrm{~K}^{-1}\right)$ \\
\hline$A$ & surface area $\left(\mathrm{m}^{2}\right)$ & $j$ & cooling parameter \\
\hline$A_{n}$ & $\mathrm{n}^{\text {th }}$ term in heat transfer equation solution & $\mathrm{J}_{0}(x)$ & Bessel function of the first kind, order \\
\hline$B i$ & Biot number & & \\
\hline$B_{n}$ & $\mathrm{n}^{\text {th }}$ term in heat transfer equation solution & $\mathrm{J}_{1}(x)$ & $\begin{array}{l}\text { Bessel function of the first kind, order } \\
\text { one }\end{array}$ \\
\hline$c$ & specific heat capacity $\left(\mathrm{J} \mathrm{kg}^{-1} \mathrm{~K}^{-1}\right)$ & $k$ & thermal conductivity $\left(\mathrm{W} \mathrm{m}^{-1} \mathrm{~K}^{-1}\right)$ \\
\hline$C$ & cooling coefficient & $k_{m}$ & thermal conductivity of cooling medium \\
\hline$d$ & smallest dimension $(\mathrm{m})$ & & $\left(\mathrm{W} \mathrm{m}^{-1} \mathrm{~K}^{-1}\right)$ \\
\hline$E$ & equivalent heat transfer dimensionality & $N u$ & Nusselt number \\
\hline$f$ & cooling parameter & $q$ & heat transfer rate $(\mathrm{W})$ \\
\hline Fo & Fourier number & $R e$ & Reynolds number \\
\hline
\end{tabular}




$\begin{array}{llll}t & \text { temperature }\left({ }^{\circ} \mathrm{C}\right) & \alpha & \text { thermal diffusivity }\left(\mathrm{m}^{2} \mathrm{~s}^{-1}\right) \\ t_{i} & \text { initial temperature }\left({ }^{\circ} \mathrm{C}\right) & \theta & \text { time }(\mathrm{s}) \\ t_{m} & \begin{array}{l}\text { cooling/freezing medium temperature } \\ \left({ }^{\circ} \mathrm{C}\right)\end{array} & \mu_{m} & \text { dynamic viscosity }\left(\mathrm{N} \mathrm{s} \mathrm{m}^{-2}\right) \\ & \mu_{n} & \mathrm{n}^{\text {th }} \text { term in heat transfer equation solution } \\ t_{s} & \text { surface temperature }\left({ }^{\circ} \mathrm{C}\right) & \rho & \text { density }\left(\mathrm{kg} \mathrm{m}^{-3}\right) \\ U & \text { cooling medium velocity }\left(\mathrm{m} \mathrm{s}^{-1}\right) & \rho_{m} & \text { density of cooling medium }\left(\mathrm{kg} \mathrm{m}^{-3}\right) \\ Y & \text { fractional unaccomplished temperature } & \phi & \text { excess temperature, } \phi=t-t_{m} \\ & \text { difference } & \omega & \text { first root of transcendental Equation }(18) \\ Z & \text { coordinate direction }(\mathrm{m}) & & \end{array}$

\section{$\underline{\text { References }}$}

1. I. Dincer, Energy 18, 335 (1993).

2. A. Alhamdan, S.K. Sastry, and J.L. Blaisdell, Trans. ASAE 33, 620 (1990).

3. F.A. Ansari, Int. Commun. Heat Mass 14, 229 (1987).

4. S.L. Chen, A.I. Yeh, and J.S.B. Wu, J. Food Eng. 31, 473 (1997).

5. B.L. Clary, G.L. Nelson, and R.E. Smith, Trans. ASAE 11, 496 (1968).

6. A.C. Cleland and R.L. Earle, Bulletin De L'Institut International Du Froid 1976-1, 361 (1976).

7. J.D. Daudin and M.V.L. Swain, J. Food Eng. 12, 95 (1990).

8. I. Dincer, Int. Commun. Heat Mass 18, 431 (1991).

9. I. Dincer, M. Yildiz, M. Loker, and H. Gun, Int. J. Food Sc. Tech. 27, 347 (1992).

10. I. Dincer, Int. Commun. Heat Mass 21, 307 (1994).

11. I. Dincer, Int. J. Heat Mass Transfer 37, 2781 (1994).

12. I. Dincer, J. Heat Trans. - T. ASME 116, 764 (1994).

13. I. Dincer and O.F. Genceli, Int. J. Heat Mass Transfer 37, 625 (1994).

14. I. Dincer, Int. J. Energy Res. 19, 95 (1995).

15. I. Dincer, Int. J. Energy Res. 19, 199 (1995).

16. I. Dincer, Int. J. Energy Res. 19, 791 (1995).

17. I. Dincer, Determination of Heat Transfer Coefficients for Spherical Objects in Immersing Experiments Using Temperature Measurements, in N. Zabaras, K.A. Woodbury, and M. 
Raynaud, (eds.), Inverse Problems in Engineering: Theory and Practice, pp. 237-241, American Society of Mechanical Engineers, New York (1995).

18. I. Dincer and O.F. Genceli, Int. J. Energy Res. 19, 205 (1995).

19. I. Dincer and O.F. Genceli, Int. J. Energy Res. 19, 219 (1995).

20. I. Dincer, Int. J. Energy Res. 20, 419 (1996).

21. I. Dincer and S. Dost, Int. J. Energy Res. 20, 587 (1996).

22. I. Dincer, J. Food Eng. 31, 59 (1997).

23. E.S. Flores and R.H. Mascheroni, J. Food Sci. 53, 1872 (1988).

24. R.L. Frederick and F. Comunian, Int. Commun. Heat Mass 21, 447 (1994).

25. A. Khairullah and R.P. Singh, Int. J. Refrig. 14, 176 (1991).

26. A. Kondjoyan and J.D. Daudin, J. Food Eng. 32, 225 (1997).

27. I. Kopelman, J.L. Blaisdell, and I.J. Pflug, ASHRAE Trans. 72, 209 (1966).

28. S. Mankad, K.M. Nixon, and P.J. Fryer, J. Food Eng. 31, 9 (1997).

29. R.E. Smith, A.H. Bennett, and A.A. Vacinek, Trans. ASAE 14, 44 (1971).

30. W.E. Stewart, B.R. Becker, M.E. Greer, and L.A. Stickler, ASHRAE Trans. 96, 142 (1990).

31. A. Vazquez and A. Calvelo, J. Food Process Eng. 4, 53 (1980).

32. A. Vazquez and A. Calvelo, J. Food Sci. 48, 114 (1983).

33. P. Verboven, B.M. Nicolaï, N. Scheerlink, and J. De Baerdemaeker, J. Food Eng. 33, 15 (1997).

34. C.A. Zuritz, S.C. McCoy, and S.K. Sastry, J. Food Eng. 11, 159 (1990).

35. J. Arce and V.E. Sweat, ASHRAE Trans. 86, 235 (1980).

36. A.C. Cleland, Food Refrigeration Processes: Analysis, Design and Simulation, Elsevier Science Publishers, London (1990).

37. H.S. Carslaw and J.C. Jaeger, Conduction of Heat in Solids, $2^{\text {nd }}$ ed., Oxford University Press, London (1980).

38. A.C. Cleland and R.L. Earle, Int. J. Refrig. 5, 98 (1982).

39. Z. Lin, A.C. Cleland, G.F. Serrallach, and D.J. Cleland, Refrig. Sci. Tech. 1993-3, 259 (1993).

40. Z. Lin, A.C. Cleland, D.J. Cleland, and G.F. Serrallach, Int. J. Refrig. 19, 95 (1996).

41. Z. Lin, A.C. Cleland, D.J. Cleland, and G.F. Serrallach, Int. J. Refrig. 19, 107 (1996). 